\title{
Access to cancer care in northwestern Ontario-a population-based study using administrative data
}

\author{
M. Febbraro $\mathrm{MD}_{1}^{*}$ M. Conlon $\mathrm{PhD}_{1}^{\dagger} \mathrm{J}$. Caswell $\mathrm{PhD}_{1}^{\dagger}$ and N. Laferriere $\mathrm{MD} \mathrm{PhD}^{\ddagger}$
}

\begin{abstract}
Background Despite universal access to health care in Canada, there are disparities relating to social determinants of health that contribute to discrepancies between rural and urban areas in cancer incidence and outcomes. Given that Canada has one of the highest-quality national population-based cancer registry systems in the world and that little information is available about cancer statistics specific to northwestern Ontario, the purpose of the present study was to estimate the percentage of cancer patients without documentation of a specialist consultation (medical or radiation oncology consultation) and to determine factors that affect access to specialist consultation in northwestern Ontario.
\end{abstract}

Methods This population-based retrospective study used administrative data obtained through the Ontario Cancer Data Linkage Project. For each index case, a timeline was constructed of all Ontario Health Insurance Plan billing codes and associated service dates, starting with the primary cancer diagnosis and ending with death. Specific factors affecting access to specialist consultation were assessed.

Results Within the 6-year study period (2010-2016), 2583 index cases were identified. Most $(n=2007,78 \%)$ received a specialist consultation. Factors associated with not receiving a specialist consultation included older age $[p<0.0001$; odds ratio (OR): $0.29 ; 95 \%$ confidence interval (CI): 0.19 to 0.44 ] and rural residence ( $p<0.0001$; OR: $0.48 ; 95 \%$ CI: 0.48 to 0.72 ). Factors associated with receiving a specialist consultation included a longer timeline ( $p<0.0001 ;$ OR: $1.32 ; 95 \%$ CI: 1.19 to 1.46 ), a diagnosis of breast cancer ( $p<0.0001$; OR: 2.51; $95 \%$ CI: 1.43 to 4.42 ), and a diagnosis of lung cancer ( $p<0.0001$; OR: 1.77 ; $95 \%$ CI: 1.38 to 2.26 ).

Conclusions This study is the first to look at care access in northwestern Ontario. The complexity and multidisciplinary nature of cancer care makes the provision of appropriate care a challenge; a one-size-fits-all disease prevention and treatment strategy might not be appropriate.

Key Words Health services accessibility, cancer care access, rural cancer services, North West LHIN, medical oncology consultation, radiation oncology consultation

\section{INTRODUCTION}

Almost half of all Canadians will develop cancer in their lifetime, with one quarter expected to die of the disease ${ }^{1}$. It is estimated that, by 2032, the number of cancer cases will be $79 \%$ higher than they were in $2007^{1}$. As a result, the Canadian health care system is expected to face an increasing demand for cancer services. It was estimated that 90,483 new cases of cancer would be diagnosed in Ontario in 2018, resulting in an age-standardized incidence of 571.1 cases per 100,000 people $^{2}$. Approximately $15 \%$ of Ontario's population lives in remote and rural areas ${ }^{1}$. After adjusting for age distribution, northwestern Ontario has significantly higher rates of colorectal cancer and lung cancer ${ }^{3}$.

In Canada, despite universal access to health care, there are disparities in income, rural or urban residence, and immigration status. Those disparities contribute to differences in cancer risk factors, screening and screening use behaviours, diagnostic and treatment service provision, and diagnostic and treatment service use ${ }^{2,4}$. Rural and 
urban areas show discrepancies in cancer incidences and outcomes for several malignancies, with poorer survival related to advanced stage at diagnosis ${ }^{5}$. For all cancers, the 2015 age-standardized mortality rate was significantly higher in northwestern Ontario than in the province overall (238 vs. 185 per 100,000 population $)^{3}$.

The North West Local Health Integration Network (LHIN) faces a unique challenge in providing optimal medical care, given its large geographic area $\left(385,000 \mathrm{~km}^{2}\right)^{6}$. Of 14 LHINs in Ontario, the North West LHIN is the largest, having a landmass the size of France ${ }^{6}$. The North West LHIN also has the lowest population density ( 0.4 people per square kilometer) ${ }^{6}$. It is home to 32 remote First Nations communities, most of which do not have road access. In 2016, the population of those communities constituted $24.1 \%$ of the total North West LHIN population ${ }^{6}$. In addition, $45 \%$ of the entire population of the North West LHIN is concentrated in a single urban setting (Thunder Bay), leaving approximately $30 \%$ of the population concentrated outside that major urban setting with its road access ${ }^{6}$.

Low population density and large geographic landmass make access to specialty care a challenge for patients in northwestern Ontario ${ }^{6}$. Individuals living outside the main urban centre often travel to Thunder Bay, Ontario, or Winnipeg, Manitoba, to access specialized services ${ }^{6}$. Patients with a diagnosis of cancer receive care through Regional Cancer Care Northwest (https://www. cancercareontario.ca/en/cancer-care-ontario/programs/ regional-cancer-programs/north-west), which is a fullservice regional cancer program providing prevention and screening services, diagnostic services, cancer treatment, survivorship services, and palliative care services ${ }^{7}$. In addition to a regional centre, it has 13 satellite chemotherapy clinics in partnering hospitals, which also have the capability to provide diagnostic services. Compared with residents of the rest of the province, residents of the North West LHIN have a lower life expectancy at birth and poorer perceived general health. The population also shows higher rates of frequent alcohol use, obesity, and daily smoking, and a lower rate of individuals with a regular physician ${ }^{3}$.

Given that Canada has one of the highest-quality national population-based cancer registry systems in the world, little information is available about cancer statistics specific to northwestern Ontario. The purpose of the present study was therefore to estimate the percentage of cancer patients without documentation of a medical or radiation oncology consultation ("specialist consultation") and to determine the factors that affect access to specialist consultation.

\section{METHODS}

The study was approved by the Research Ethics Board of the Thunder Bay Regional Health Sciences Centre and Lakehead University.

This population-based retrospective study used administrative data. The study cohort was defined as all residents of the North West LHIN with a pathologic diagnosis of any cancer who died from any cause between 2010 and 2016. In consultation with the care team at the Thunder Bay Regional Cancer Program, the authors approved a dataset beginning in 2010. A decedent cohort was used to ensure that a start and end to index case timelines could be established in their entirety without any timeline continuing beyond the study period.

Data for the cohort were obtained through the Ontario Cancer Data Linkage Project, a cancer data release mechanism established in 2008 as a joint initiative of the Ontario Institute for Cancer Research, Ontario Health (Cancer Care Ontario), and ICES. The study variables were chosen with the help of ICES such that all data could be accounted for in full without having to adjust for missing data. After completion of a data use agreement, the authors were provided with risk-reduced de-identified data.

The Ontario Cancer Registry, a provincial database for all Ontario residents diagnosed with cancer, was the source for the identification of index cases. Using encrypted provincial health card numbers, the index cases were additionally linked to the OHIP (Ontario Health Insurance Plan) claims database, the Registered Persons Database, and Statistics Canada's Community Profiles.

For each index case, a timeline of all OHIP billing codes and associated service dates was constructed, beginning with the date of the primary cancer diagnosis and ending with the date of death. "Cancer diagnosis" was defined as the date of pathologic diagnosis. Ontario Health (Cancer Care Ontario) reports that the average wait time for cancer specialist consultation is approximately 4 weeks. Given that average, timelines of less than 30 days were removed from the study, because those patients would have been outside the average waiting period. All remaining timelines were assessed to see whether a specialist consultation occurred before the date of death. Specialist consultation was estimated using the presence of at least 1 of the following OHIP billing codes at any point in the timeline: A135, A136, A345, A435, A346, A445, A446, A615, A765, A845, C135, C136, C345, C346, C435, C445, C446, C615, C745, C765, C845.

For the study cohort, the Ontario Cancer Registry provided the number of primary cancers, the date of the primary cancer diagnosis, date of death, and year of death. Timeline duration was calculated as the number of days from the primary diagnosis to death. Age (stratified into 7 categories, with the youngest age category being 40-54 years) and sex were obtained from the Registered Persons Database. Income quintile was estimated using Statistics Canada's Postal Code Conversion File Plus to link median income from the Community Profiles database to the patient's postal code in the Registered Persons Database. Rural or urban residence was defined using Statistics Canada's census definition of rural, which is the population outside settlements of 1000 or more population with a population density of 400 or more inhabitants per square kilometer. The Deyo adaptation of the Charlson comorbidity index is an index commonly used in health services research to capture the outcome effect of any of 22 diseases that a patient might have in addition the disease of interest (in this case, cancer). Each of the diseases is assigned a value, and the sum of the values produces a Charlson-Deyo comorbidity index. That index value was provided as part of the data acquisition process through ICES, with a lookback period of 20 years.

Descriptive statistics and frequencies were calculated for all covariates, stratified by cohort (ever- and 
never-consultation groups). Unadjusted differences between cohorts were determined using the chi-square test for categorical variables and the 2-sample Wilcoxon test for duration of disease. Univariate and multivariate logistic regression analyses were conducted to calculate, respectively, crude (unadjusted) and adjusted odds ratios (ORs) with their 95\% confidence intervals (CIs). Covariates were adjusted based on a literature review that determined their relevance to whether an index case would be likely to receive a certain treatment. Results were considered statistically significant at $p<0.05$. All analyses were performed using the SAS software application (version 9.4: SAS Institute, Cary, NC, U.S.A.).

\section{RESULTS}

The data search identified 2583 index cases with a primary cancer diagnosis and a date of death between 2010 and 2016. Index cases diagnosed more than 30 days before death were included and are reflected in that total. In most cases ( $n=2007,78 \%$ ), the patient had a specialist consultation. Common primary diagnoses included lung cancer $(n=673$, $26.1 \%)$, colon cancer $(n=265,10.3 \%)$, prostate cancer $(n=$ $140,5.4 \%)$, and breast cancer ( $n=137,5.3 \%$; Table I). The median timeline was $0.51 \pm 1.14$ years in the no consultation cohort and $1.33 \pm 1.53$ years in the consultation cohort.

Multivariable logistic regression identified 5 significant and independent predictors that affected the likelihood of having a specialist consultation (Table II). Patients who were more than 80 years of age $(p<0.0001$; OR: $0.29 ; 95 \%$ CI: 0.19 to 0.44$)$ or who resided in rural location ( $p<0.0001$; OR: 0.48 ; $95 \%$ CI: 0.48 to 0.72 ) were less likely to receive specialist consultation. Patients with a longer timeline ( $p<0.0001$; OR: 1.32 ; 95\% CI: 1.19 to 1.46 ), a diagnosis of breast cancer ( $p<0.0001$; OR: 2.51 ; $95 \%$ CI: 1.43 to 4.42 ), or a diagnosis of lung cancer ( $p<0.0001$; OR: 1.77 ; 95\% CI: 1.38 to 2.26 ) were more likely to have a specialist consultation.

Variables assessed that did not affect the likelihood of receiving a specialist consultation were sex, Charlson-Deyo comorbidity index, income quintile, and year of death.

No data were missing in the analysis, and none of the foregoing results had to be adjusted for missing information.

\section{DISCUSSION}

We found that most patients with a primary cancer diagnosis who died from any cause between 2010 and 2016 (78\%) received a specialist consultation. We are not aware of any other study or published document that provides this information for northwest Ontario. Our study is also the first to have looked at access to cancer care services as they relate to consultation through an analysis of administrative data for a decedent cohort.

With respect to variables contributing to access to care, our study strays from the published literature ${ }^{7}$, with some similarities noted. Four variables are commonly implicated in social disparities: socioeconomic status, sex, ethnicity, and geographic area. In addition, age is an additional variable commonly implicated when studying equity in cancer care ${ }^{7}$. No statistical significance was observed when
TABLE I Characteristics of identified index cases with a primary cancer diagnosis and a date of death during 2010-2016

\begin{tabular}{|c|c|c|c|}
\hline \multirow[t]{2}{*}{ Covariate } & \multicolumn{2}{|c|}{ Specialist consultation } & \multirow{2}{*}{$\begin{array}{c}p \\
\text { Value }\end{array}$} \\
\hline & No & Yes & \\
\hline Patients ( $n$ ) & 576 & 2007 & \\
\hline $\operatorname{Sex}[n(\%)]$ & & & 0.214 \\
\hline Men & $300(52.08)$ & $1104(55.01)$ & \\
\hline Women & $276(47.92)$ & $903(44.99)$ & \\
\hline Income quintile $[n(\%)]$ & & & 0.121 \\
\hline 1 (lowest) & $154(26.74)$ & $453(22.57)$ & \\
\hline 2 & $105(18.23)$ & 356 (17.74) & \\
\hline 3 & $103(17.88)$ & $431(21.47)$ & \\
\hline 4 & $106(18.40)$ & $410(20.43)$ & \\
\hline 5 (highest) & $108(18.75)$ & 357 (17.79) & \\
\hline Rural $[n(\%)]$ & & & $<0.0001$ \\
\hline No & 343 (59.55) & 1385 (69.01) & \\
\hline$Y_{e s}{ }^{a}$ & $233(40.45)$ & $622(30.99)$ & \\
\hline Age group [n (\%)] & & & $<0.0001$ \\
\hline 40-54 Years & $33(5.73)$ & $211(10.51)$ & \\
\hline 55-59 Years & $36(6.25)$ & $170(8.47)$ & \\
\hline 60-64 Years & $51(8.85)$ & $246(12.26)$ & \\
\hline 65-69 Years & $55(9.55)$ & $257(12.81)$ & \\
\hline 70-74 Years & $75(13.02)$ & $285(14.20)$ & \\
\hline 75-79 Years & $67(11.63)$ & $323(16.09)$ & \\
\hline$\geq 80$ Years & $259(44.97)$ & $515(25.66)$ & \\
\hline Year of death $[n(\%)]$ & & & 0.134 \\
\hline 2010 & $35(6.08)$ & $120(5.98)$ & \\
\hline 2011 & $103(17.88)$ & $286(14.25)$ & \\
\hline 2012 & $98(17.01)$ & $308(15.35)$ & \\
\hline 2013 & $89(15.45)$ & $360(17.94)$ & \\
\hline 2014 & 89 (15.45) & 344 (17.14) & \\
\hline 2015 & $118(20.49)$ & $390(19.43)$ & \\
\hline 2016 & $44(7.64)$ & 199 (9.92) & \\
\hline Cancer type $[n(\%)]$ & & & $<0.0001$ \\
\hline Breast & $16(2.78)$ & $121(6.03)$ & \\
\hline Lung & $114(19.79)$ & $559(27.85)$ & \\
\hline Colorectal & $73(12.67)$ & $192(9.57)$ & \\
\hline Prostate & $31(5.38)$ & $109(5.43)$ & \\
\hline Other & $342(59.38)$ & $1026(51.12)$ & \\
\hline Deyo-CCI score $[n(\%)]$ & & & 0.0006 \\
\hline 0 & $406(70.49)$ & $1567(78.08)$ & \\
\hline 1 & $70(12.15)$ & $170(8.47)$ & \\
\hline $2+$ & $100(17.36)$ & $270(13.45)$ & \\
\hline $\begin{array}{r}\text { Timeline [Dx to } \\
\text { death (years)] }\end{array}$ & & & $<0.0001$ \\
\hline Median \pm IQR & $0.51 \pm 1.14$ & $1.33 \pm 1.53$ & \\
\hline
\end{tabular}

a As defined by Statistics Canada, 2007.

Deyo- $\mathrm{CCl}=$ Deyo modification of the Charlson comorbidity index; $\mathrm{Dx}=$ diagnosis; $\mathrm{IQR}=$ interquartile range.

assessing for income quintile and sex, but older age and rural location were found to be associated with whether a patient received a specialist consultation.

Index cases in which the patient was more than 80 years of age were less likely to include a specialist 
TABLE II Univariable and multivariable logistic regression for independent predictors of the likelihood of receiving specialist consultation

\begin{tabular}{|c|c|c|c|c|}
\hline \multirow[t]{2}{*}{ Variable } & \multicolumn{2}{|c|}{ Crude result } & \multicolumn{2}{|c|}{ Adjusted resulta } \\
\hline & OR & $95 \% \mathrm{Cl}$ & OR & $95 \% \mathrm{Cl}$ \\
\hline \multicolumn{5}{|l|}{ Rural } \\
\hline No & \multicolumn{2}{|c|}{ Reference } & \multicolumn{2}{|c|}{ Reference } \\
\hline Yes & 0.66 & 0.55 to 0.80 & 0.58 & 0.48 to 0.72 \\
\hline \multicolumn{5}{|l|}{ Age group } \\
\hline 40-54 Years & \multicolumn{2}{|c|}{ Reference } & \multicolumn{2}{|c|}{ Reference } \\
\hline 55-59 Years & 0.74 & 0.44 to 1.23 & 0.68 & 0.40 to 1.14 \\
\hline $60-64$ Years & 0.75 & 0.47 to 1.21 & 0.71 & 0.44 to 1.15 \\
\hline 65-69 Years & 0.73 & 0.46 to 1.17 & 0.65 & 0.40 to 1.05 \\
\hline 70-74 Years & 0.59 & 0.39 to 0.93 & 0.54 & 0.34 to 0.86 \\
\hline 75-79 Years & 0.75 & 0.48 to 1.18 & 0.64 & 0.40 to 1.03 \\
\hline$\geq 80$ Years & 0.31 & 0.21 to 0.46 & 0.29 & 0.19 to 0.44 \\
\hline \multicolumn{5}{|l|}{ Cancer type } \\
\hline Other & \multicolumn{2}{|c|}{ Reference } & \multicolumn{2}{|c|}{ Reference } \\
\hline Breast & 2.52 & 1.48 to 4.31 & 2.51 & 1.43 to 4.42 \\
\hline Lung & 1.64 & 1.29 to 2.07 & 1.77 & 1.38 to 2.26 \\
\hline Colorectal & 0.88 & 0.65 to 1.18 & 0.90 & 0.66 to 1.23 \\
\hline Prostate & 1.17 & 0.77 to 1.78 & 1.0 & 0.63 to 1.57 \\
\hline Timeline (Dx to death) & 1.30 & 1.19 to 1.42 & 1.32 & 1.19 to 1.46 \\
\hline
\end{tabular}

a Adjusted for all variables.

$\mathrm{OR}=$ odds ratio; $\mathrm{Cl}=$ confidence interval; $\mathrm{Dx}=$ diagnosis.

consultation, which could mean that patients in that age group are not equipped to deal with the travel required to obtain one. Studies have noted that access to specialist care is limited for rural patients of advanced age $\mathrm{e}^{8-12}$. Treatment and disease burden can make it difficult for patients to remain mobile, let alone travel for treatment. Several studies have found that increased travel time affects patient-care decisions specific to cancer ${ }^{10,13,14}$. The most specific of those studies found that travelling more than 1 hour to treatment was associated with increased unmet patient needs ${ }^{15}$. Outreach clinics, virtual tumour boards, tele-oncology, and other telemedicine applications have been identified as being pivotal in improving care access in the rural setting, especially with an aging population $^{16}$. Telemedicine videoconference consultations are used throughout the North West LHIN, with increased implementation known to benefit patients specifically in northwestern Ontario ${ }^{17}$.

In its 2016-2017 annual report, the North West LHIN noted that the region's landmass and dispersed population presented a unique challenge to health service delivery, which included access to care, health human resources, and the need for extensive travel. The result is higher health care costs per capita ${ }^{6}$. Rurality is considered a type of vulnerability ${ }^{13}$. Geographic distance from services poses access barriers, which are worsened by transportation problems or weather conditions ${ }^{13}$. Travel in northwestern Ontario can be challenging, especially in the winter months. The additional burden of weather on a population with lesser road access might impose additional access barriers. Unfortunately, in the present study, we did not assess variables such as month of diagnosis or distance from the nearest cancer centre, which could help to answer that hypothesis-generating concern.

As pertains to breast cancer, worse survival in rural regions appears to be related to advanced stage at diagnosis ${ }^{18}$. A discrepancy in cancer stage at diagnosis in rural-residing compared with urban-residing patients is related to rural residence being associated with significantly different referral practices, leading to an effect on treatment outcomes and overall health outcomes ${ }^{19}$. Unfortunately, because of limitations in the administrative dataset, we were unable to identify stage at cancer diagnosis. The dataset was incomplete for the period studied, which is a limitation, given that early-stage cancers might not require referral to medical or radiation oncology, a factor not captured in the study.

In the present study, we did not assess patient views or the rationale behind not receiving a specialist consultation, which will be a topic for future study. Patients referred for specialist consultation but not receiving a visit could not be captured with the use of an administrative dataset. Factors contributing to a patient not going forward with a specialist referral could not be assessed in the present study, but are documented in the literature. In one study, health and social data were linked to demonstrate how social determinants are associated with primary care service provision ${ }^{20}$. Increased social complexity was strongly associated with poorer outcomes in primary care indicators for prevention, chronic disease management, geriatric care, and use of health services ${ }^{20}$. It would be interesting to link the data used in our project with primary care data to see if further disparities related to cancer care in northwest Ontario could be ascertained, thus reflecting primary care needs as they relate to cancer. 
Our study was approved at a time when the North West LHIN planned to change its telemedicine scheduling practices, beginning with Regional Cancer Care Northwest. The intention was to provide integrated scheduling such that the service provider is responsible for scheduling tasks, with a goal of accommodating scheduling of up to 5000 additional events per year ${ }^{21}$. With changes to the LHIN structure fast approaching, consideration of geographic distribution and access to cancer services in northwest Ontario will be integral to the ongoing success of cancer care in the North ${ }^{22}$.

\section{CONCLUSIONS}

Canada shows geographic variation in cancer incidence rates secondary to differences in modifiable risk factors such as unhealthy diet, smoking, obesity, and physical inactivity ${ }^{3}$. Rural patients with lower health literacy might feel culturally marginalized in an urban health care context, which might lessen a patient's willingness to receive distant health care ${ }^{1}$.

The complexity and multidisciplinary nature of cancer treatment make the provision of appropriate care a challenge, necessitating a clear understanding of where along the continuum of care inequality occurs. In addition, remote areas might need special attention in terms of policy implications; a one-size-fits-all disease prevention and treatment strategy might not be appropriate.

\section{ACKNOWLEDGMENTS}

This study was supported through provision of data by ICES and Ontario Health (Cancer Care Ontario) [OH(CCO)] which receive funding support in an annual grant from the Ontario Ministry of Health and the Ontario Institute for Cancer Research (OICR). The opinions, results, and conclusions reported in this paper are those of the authors. No endorsement by ICES, OH(CCO), OICR, or the Ontario Ministry of Health is intended or should be inferred.

\section{CONFLICT OF INTEREST DISCLOSURES}

We have read and understood Current Oncology's policy on disclosing conflicts of interest, and we declare that we have none.

\section{AUTHOR AFFILIATIONS}

*Northern Ontario School of Medicine, McMaster University, Thunder Bay; 'Institute for Clinical Evaluative Sciences North, and Epidemiology, Outcomes and Evaluation Research, Health Sciences North Research Institute, Northeast Cancer Centre, Sudbury; and ${ }^{\ddagger}$ Thunder Bay Regional Health Sciences Centre, Thunder Bay, ON.

\section{REFERENCES}

1. Canadian Cancer Society's Advisory Committee on Cancer Statistics. Canadian Cancer Statistics 2017. Toronto, ON: Canadian Cancer Society; 2017.

2. Ontario Health (Cancer Care Ontario) $[\mathrm{OH}(\mathrm{CCO})]$. Ontario Cancer Statistics 2018. Toronto, ON: OH(CCO); 2018.

3. North West LHIN. Population Health Profile. Thunder Bay, ON: Queen's Printer for Ontario; 2015. [Downloadable from:http:// www.northwestlhin.on.ca/ForHealthServiceProviders/ North\%20West\%20LHIN\%20Population\%20Health\%20 Profiles.aspx; cited 8 June 2020]

4. Kerner J, Liu J, Wang K, et al. Canadian cancer screening disparities: a recent historical perspective. Curr Oncol 2015; 22:156-63.
5. Hallet J, Law CH, Karanicolas PJ, Saskin R, Liu N, Singh S. Rural-urban disparities in incidence and outcomes of neuroendocrine tumors: a population-based analysis of 6271 cases. Cancer 2015;121:2214-21.

6. North West Local Health Integration Network (LHIN). Advancing the Future of Health Care: North West LHIN Annual Report 2016-2017. Thunder Bay, ON: North West LHIN; 2017.

7. Sinding W, Warren R, Fitzpatrick-Lewis D, Sussman J. Research in cancer care disparities in countries with universal healthcare: mapping the field and its conceptual contours. Support Care Cancer 2014;22:3101-20.

8. Conlon M, Hartman M, Ballantyne B, Aubin N, Meigs M, Knight A. Access to oncology consultation in a cancer cohort in northeastern Ontario. Curr Oncol 2015;22:e69-75.

9. Groome PA, Schulze KM, Keller S, Mackillop WJ. Demographic differences between cancer survivors and those who die quickly of their disease. Clin Oncol (R Coll Radiol) 2008;20:647-56.

10. Maddison AR, Asada Y, Urquhart R, Johnstone G, Burge F, Porter G. Inequity in access to guideline-recommended colorectal cancer treatment in Nova Scotia, Canada. Healthc Policy 2012;8:71-87.

11. Steyerberg EW, Neville B, Weeks JC, Earle CC. Referral patterns, treatment choices, and outcomes in locoregional esophageal cancer: a population-based analysis of elderly patients. J Clin Oncol 2007;25:2389-96.

12. Ward MM, Ullrich F, Matthews K, et al. Who does not receive treatment for cancer? J Clin Oncol 2013;9:20-6.

13. Brundisini F, Giacomini M, DeJean D, Vanstone M, Winsor S, Smith A. Chronic disease patients' experiences with accessing health care in rural and remote areas: a systematic review and qualitative meta-synthesis. Ont Health Technol Assess Ser 2013;13:1-33.

14. Lavergne MR, Johnston GM, Gao J, Dummer TJ, Rheaume DE. Variation in the use of palliative radiotherapy at end of life: examining demographic, clinical, health service, and geographic factors in a population-based study. Palliat Med 2011;25:101-10.

15. Tzelepis F, Paul CL, Sanson-Fisher RW, et al. Unmet supportive care needs of haematological cancer survivors: rural versus urban residents. Ann Hematol 2018;97:1283-92.

16. Charlton M, Schlichting J, Chioreso C, Ward M, Vikas P. Challenges of rural cancer care in the United States. Oncology (Williston Park) 2015;29:633-40.

17. Mashru J, Kirlew M, Saginur R, Schreiber YS. Management of infectious diseases in remote northwestern Ontario with telemedicine videoconference consultations. J Telemed Telecare 2017;23:83-7.

18. Nguyen-Pham S, Leung J, McLaughlin D. Disparities in breast cancer stage at diagnosis in urban and rural adult women: a systematic review and meta-analysis. Ann Epidemiol 2014;24:228-35.

19. Johnson AM, Hines RB, Johnson JA 3rd, Bayakly AR. Treatment and survival disparities in lung cancer: the effect of social environment and place of residence. Lung Cancer 2014; 83:401-7.

20. Katz A, Chateau D, Enns JE, et al. Association of the social determinants of health with quality of primary care. Ann Family Med 2018;16:217-24.

21. North West Local Health Integration Network (LHIN). North West LHIN Annual Business Plans [Web page]. Thunder Bay, ON: North West LHIN; n.d. [Available at: http://www. northwestlhin.on.ca/accountability/annualbusinessplan. aspx; cited 1 March 2019]

22. Ward MM, Ullrich F, Matthews K, et al. Access to chemotherapy services by availability of local and visiting oncologists. J Oncol Pract 2014;10:26-31. 